\title{
Distributed Energy Management of PV-Storage Systems for Voltage Rise Mitigation
}

\author{
Bananeh Ansari $^{1}$ (D) . Marcelo G. Simoes ${ }^{1}$
}

Received: 18 August 2016 / Accepted: 28 August 2017 / Published online: 8 September 2017

(C) Springer Nature Singapore Pte Ltd. 2017

\begin{abstract}
This paper develops a distributed consensusbased energy management scheme (EMS) for multiple photovoltaics+energy storage systems (PV+ESS) connected to a smart distribution network. First, each customer individually determines the optimal size and initial scheduling of ESS to be installed in parallel with PV over a dayahead planning horizon. The objective of installing ESS is to reduce the electricity bill while minimizing ESS aging effect. Then, after the day-ahead planning, customers manage their system in a near real-time fashion to account for voltage conditions in order to avoid voltage-rise-associated curtailments. Customers along the feeder communicate with each other and exchange information about voltage deviation at their nodes in order to reach a consensus about average voltage situation in the distribution network. Based on voltage situation, EMS updates the lower bound on net power exchange with the grid for the hour ahead. Also, an iterative method is proposed to determine the lifetime of ESS and the economic benefits gained over the lifetime. Simulation results for IEEE 33-bus test system prove the effectiveness of the proposed EMS and its financial viability.
\end{abstract}

Keywords Energy management · Energy storage · Distribution networks · Photovoltaics - Voltage rise mitigation

Bananeh Ansari

bansari@mines.edu

11600 Illinois St., Golden, CO 80401, USA

\section{Nomenclature}

Electric Power Distribution System

$t \quad$ Time step [h] for day-ahead planning, [yr] for economic analysis

$T \quad$ Planning horizon [h]

$\pi_{t} \quad$ Electricity price $[\$ / \mathrm{kWh}]$

$\lambda$ Unit cost of storage $[\$ / \mathrm{kWh}]$

$\zeta^{B} \quad$ Aging coefficient

$\eta^{B} \quad$ Battery round-trip efficiency

$C^{B, \text { nom }} \quad$ Battery nominal capacity $[\mathrm{kWh}]$

$C_{t}^{B} \quad$ Effective battery capacity [kWh]

$\Delta C_{t}^{B} \quad$ Battery capacity degradation $[\mathrm{kWh}]$

$P_{t}^{\text {net }} \quad$ Net real power purchase from the grid [kW]

$P_{t}^{B^{+}} \quad$ Battery charge rate measured at the output of the converter [kW]

$P_{t}^{B^{-}} \quad$ Battery discharge rate measured at the output of the converter [kW]

$E_{t}^{B} \quad$ Stored energy in the battery [kWh]

$s_{t}^{B} \quad$ Battery state-of-charge (SOC)

$d^{B} \quad$ Battery minimum depth-of-discharge ( $\left.D O D\right)$

$P_{t}^{P V} \quad$ PV power generation $[\mathrm{kW}]$

$P_{t}^{D} \quad$ Real power demand $[\mathrm{kW}]$

$i \quad$ Node index

$N \quad$ Total number of nodes in the distribution network

$V_{\max } \quad$ Maximum allowable voltage [p.u.]

$V_{t, i} \quad$ Node voltage [p.u.]

$\delta V_{t, i} \quad$ Voltage deviation [p.u.]

$\delta \bar{V}_{t} \quad$ Average voltage deviation from [p.u.]

Multi-agent System

$k \quad$ Dynamic system time step

$i \quad$ Agent index 
$\mathcal{G} \quad$ Graph representation of the MAS network

$L^{\mathcal{G}} \quad$ Graph-Laplacian of $\mathcal{G}$

$\Phi \quad$ Perron matrix of graph $\mathcal{G}$

$B_{j} \quad$ Set of the neighboring (adjacent) agents of agent $j$

$x_{k, i} \quad$ State of the agent

$x_{0, i} \quad$ Initial state of the agent

$\epsilon \quad$ Updating parameter

$\mathbf{x}_{k} \quad$ State vector

\section{Introduction}

Integration of distributed energy resources into low- and medium-voltage distribution networks is growing to a significant level. Solar photovoltaic (PV) comprises a large portion of distributed generation connected to distribution networks. Significant "reverse" power flow is a common issue in networks with high PV penetration. When PV generation is larger than local consumption, excess power will flow upstream toward the substation, creating a "negative" voltage drop (or, equivalently, a voltage rise) along the feeder. Voltage rise is particularly significant on long feeders with PV at the end [4]. Common solutions to overcome voltage rise issue include: generation curtailment, grid reinforcement, voltage regulation using traditional voltage regulating devices including on-load tap-changing transformers (OLTC) and step voltage regulators (SVR), and reactive power provision by $\mathrm{PV}$ inverters.

Utilities widely use generation curtailment as the simplest way to alleviate overvoltage. Although effective, this approach will cause considerable generation loss, and hinders further PV integration. In grid reinforcements, a large portion of investment is underutilized for most of the new equipment's life [5]. If we use OLTC and SVRs to regulate voltage in a bidirectional distribution network, they need to switch more frequently, and this will put significant mechanical stress on the equipment, and inevitably reduce its lifetime [9, 21]. PV inverters can regulate the voltage at their point of common coupling (PCC) with the network by providing reactive power. However, under current utility practices (i.e., IEEE 1547 and UL 1741), distributed resources including PV "shall not actively regulate the voltage at their PCC" [6, 9]. Moreover, reactive power provision reduces the lifetime of PV inverters without having any financial benefits for private entities owning the PV system, unless a proper compensation mechanism exists [7].

Energy storage systems (ESS) have shown potentials for improving operating capabilities of the grid. ESS can contribute to system reliability and resiliency, and defer upgrades necessary for accommodating new installed generation [5]. An ESS used for upgrade deferral can simultaneously provide voltage support on distribution feeders. ESS can effectively mitigate voltage fluctuations with minimal real power control [1].

Deployment of new technologies on the customer side of the meter (i.e. "behind the meter") allows the customers to define their own service requirements and also contribute to grid reliability, performance and economics. Voltage regulation per se is not a service that behind-the-meter ESS are willing to provide. This is due to the fact that these ESS are privately-owned and located behind the meter, which makes them less concerned about the distribution network. However, the economic benefits of voltage regulation that can incentivize behind-the-meter ESS to manage energy exchanges with the grid such that minimal voltage fluctuations occur. When installed in parallel with a PV system (a PV+ESS), an ESS can absorb excess PV generation and prevent it from flowing into the grid, and creating voltage rise. ESS can later release the stored energy for local consumption or back injection when the price of electricity is high. This will reduce the customer's electricity bill, and shave the peak demand which, in the long run, benefit the utility.

Since PV inverters cannot regulate voltage at their PCC with grid, the easiest way to mitigate the voltage is generation curtailment. Under the revised German Renewable Energies Act of 2012, PV systems smaller than $30 \mathrm{~kW}_{p}$ either have to permanently limit their real power injection to $70 \%$ of the installed PV capacity or be equipped with remote curtailment control. PV systems with installed capacity of $30 \mathrm{~kW}_{p}$ or above must always be able to be curtailed remotely [18] (hereinafter, we call this the $70 \%$ rule).

Customers owning PV systems need to limit their injection to the grid to avoid voltage rise and consequent forced curtailment. For that purpose, it is necessary for the customers to know the relationship between real power injection at their PCCs with voltage variations at all other nodes in the distribution network. However, end-users normally do not have access to the distribution network model and measurements (except at their own PCC), and therefore, the exact relationship between real power variations and voltage fluctuations are unknown to them. If customers at various nodes are willing to share their information with the other nodes in the distribution network, all of them will have more information about the voltage situation throughout the network and hence, can manage their real power exchanges with the grid in a more optimal way. This description of multiple PV+ESS nodes complies with the properties of a multi-agent system (MAS). Each PV+ESS node can potentially be a smart agent that communicates and cooperates with other agents so that the group (i.e., the MAS) reaches an agreement on voltage situation, based on which each 
agent can decide about the amount of real power purchase from the grid.

Several literature has addressed distributed voltage regulation. Baran and El-Markabi [3] propose a control net protocol (CNP)-based MAS for dispatch and coordination of distributed energy resources (DER) for reactive power provision and voltage support. Sulc et al. [17] investigate the problem of distributed reactive power dispatch by PV inverters using consensus-based versions of alternating direction method of multipliers and dual-ascent method. Each node solves a local optimization problem for respective local copies of the global variable shared between all nodes. Ansari et al. [2] develop a method of sizing and scheduling PV+ESS in which sensitivity analysis is the basis for determining dynamic upper and lower bounds of real power exchanges with the grid in order to eliminate over- and under-voltage.

In this work, we develop a distributed agent-based energy management scheme (EMS) for multiple PV+ESS connected to a smart distribution network. The agents communicate with each other in order to reach a "consensus" about the voltage situation in the distribution network. Each agent is capable of taking voltage measurements at its PCC with the grid, communicating with its neighboring agents, and running optimization.

First, each agent individually determines the optimal size and schedule of the ESS to be installed in parallel with the PV system over a 24-hour planning horizon (i.e. day-ahead). The objective of installing ESS is reducing the electricity bill while minimizing ESS aging effect. At this stage, there is no communication between agents, and we assume that agents (regardless of installed capacity) solely rely on the $70 \%$ limit on real power injection as their voltage-regulatory constraint in the EMS sizing+scheduling optimization problem. Second, on an hourly basis, the agents measure the difference between voltage at their PCC with the grid and maximum allowable voltage. Then each agent communicates with its neighbors and shares its voltage deviation. The agents will then reach a consensus about the voltage situation in the network. It can be shown that all agents will asymptotically reach an equilibrium, which is a weighted average of their initial voltage deviations [12]. Considering the new voltage situation, the agents will re-run the EMS optimization problem (which is reduced to a schedulingonly problem) and decide to whether or not revise their previous day-ahead schedule which was based on the $70 \%$ real power injection limit (i.e. the day-ahead schedule).

In addition, an iterative framework is developed to estimate the lifetime of the ESS. This framework allows customers to analyze their economic benefits brought by ESS over the entire lifetime of it.
The contribution of this work are as follows:

- It develops a fully distributed EMS based on the notion of consensus in networked MAS. Almost all of the existing distributed EMS schemes, either rely on the decomposition of a global optimization problem into subproblems to be solved by individual agents, or on a Master-Slave MAS scheme [3, 8, 23]. In the proposed EMS, there is no global problem to solve since the objective of the agents is to minimize their own electricity bill, individually. Also, all the agents have the same situation with respect to the distribution network and its operator (i.e., the utility) so the usage of a Master-Slave scheme is not possible.

- It focuses on the behind-the-meter PV+ESS system, and therefore, we have the assumption that the agents do not have any information about the distribution network model. Thus, the EMS is model-independent.

- It uses a quite realistic model for ESS capturing the dynamics of ESS and its capacity degradation.

The organization of the rest of this paper is as follows: "Energy Management of PV+ESS" presents problem definition and formulation, "Solution Algorithm" includes the solution algorithm, "Simulation Results" provides the simulation results, and "Conclusion" finishes the paper with conclusions and future work.

\section{Energy Management of PV+ESS}

Figure 1 shows a commercial/residential customer with a $\mathrm{PV}+\mathrm{ESS}$ at the PCC with the grid. Smart meters $N, B$, and $P$ measure the net power purchase from the grid, battery charge/discharge rate, and PV power generation, respectively. Meters $N$ and $B$ are capable of net-metering meaning that they can measure bidirectional power flow. Note that in this work, ESS specifically refers to battery energy storage technology.

The system shown in Fig. 1 can have different modes of operation depending on the demand and availability of resources. For example, when PV is unavailable, the customer can discharge ESS or purchase electricity from the grid to supply the load.

The main and only purpose of using ESS in the system shown in Fig. 1 is to manage net power exchanges with the grid to reduce the electricity bill (or making profit if possible). There is always a trade-off between using ESS to save energy costs, and ESS aging. Although storage pricing is on a downward trend [5], ESS is still an expensive technology and it ages rather rapidly as it goes through charge/discharge cycles. Hence, it is important for the customer to size and 
Fig. 1 PV+ESS configuration

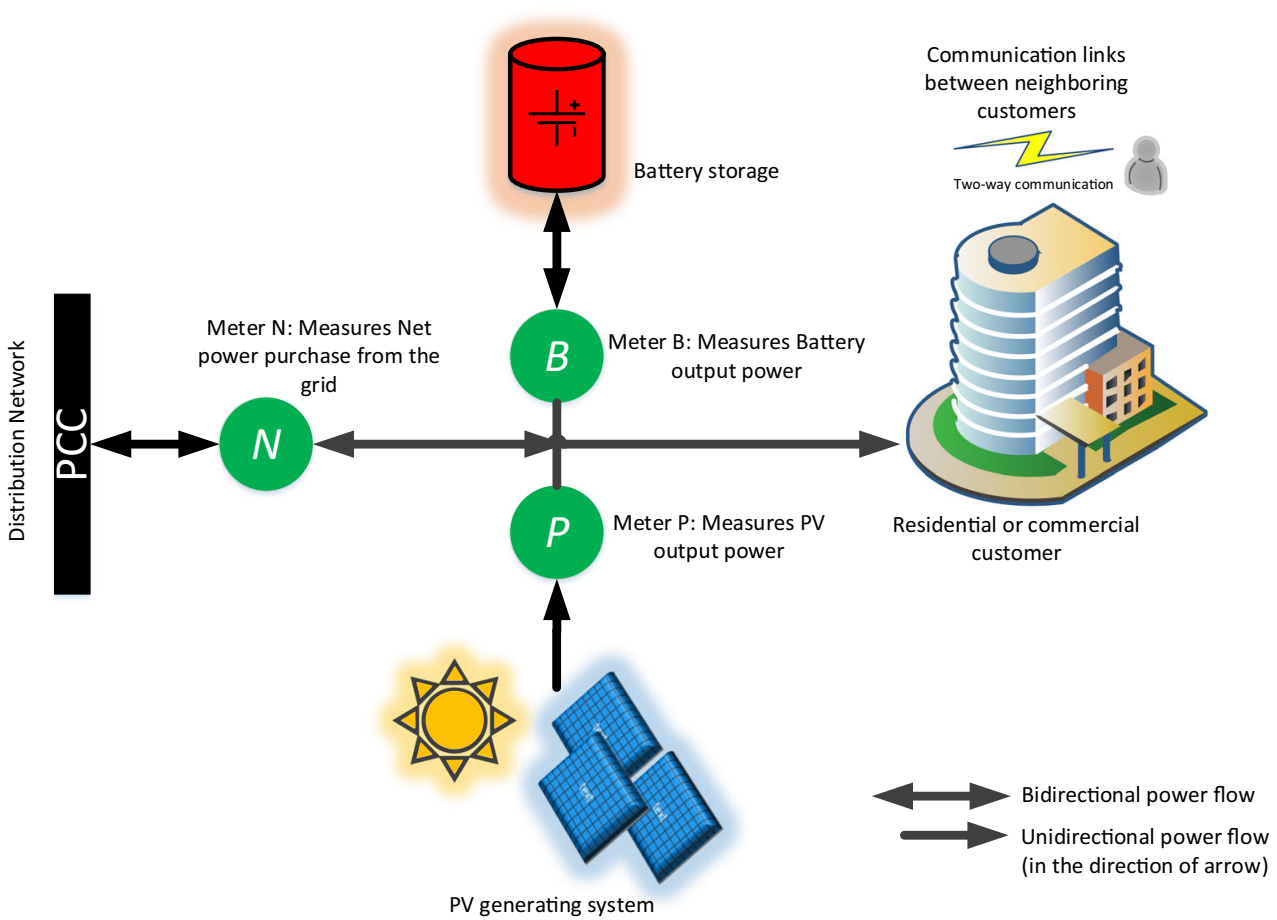

manage ESS to ensure maximum profitability and minimum aging.

For a customer, the electricity cost has two components: electricity bill and ESS aging cost. Thus, the overall objective of EMS will be:

$\min _{\mathbf{z}} \sum_{t=1}^{T} P_{t}^{n e t} \pi_{t}+\lambda \Delta C_{t}^{B}$

When $P^{\text {net }}>0$ customer purchases power from the grid, and when $P^{\text {net }}<0$, customer sells power to the grid.

Objective function (1) can be used both for sizing ESS and system scheduling. If the objective of EMS is sizing+scheduling:

$\mathbf{z}=\left[P_{1}^{n e t}, P_{2}^{n e t}, \ldots, P_{T}^{n e t}, C^{B, n o m}\right]$

$C_{1}^{B}=C^{B, \text { nom }}$

This way, customer's EMS will size simultaneously ESS and schedule power exchanges with the grid.

If ESS nominal capacity is known and the objective of EMS is only scheduling, then decision variables will reduce to

$\mathbf{z}=\left[P_{1}^{n e t}, P_{2}^{n e t}, \ldots, P_{T}^{n e t}\right]$

\section{Technical Constraints}

Objective (1) is subject to several constraints related to system operational requirements and ESS technology consideration.

At any time $t$, power balance must hold true at the PCC between the customer and the grid:

$P_{t}^{n e t}=P_{t}^{D}-P_{t}^{P V}+P_{t}^{B^{+}}-P_{t}^{B^{-}}$

Since the battery cannot charge and discharge simultaneously, in Eq. $5 P_{t}^{B^{+}}+$and $P_{t}^{B^{-}}$cannot be nonzero simultaneously. Thus,

$P_{t}^{B^{+}} \cdot P_{t}^{B^{-}}=0$

Energy conversion equation governs the dynamics of battery storage. In discrete-time domain:

$E_{t}^{B}=E_{t-1}^{B}+\eta^{B} P_{t}^{B^{+}}-\frac{P_{t}^{B^{-}}}{\eta^{B}}$

Note that, when sizing or scheduling the battery, we assume:

$E_{1}^{B}=E_{T}^{B}$

ESS cannot store more energy than the effective capacity, and also cannot be discharged to less than its minimum DOD:

$d^{B} C_{t}^{B} \leq E_{t}^{B} \leq C_{t}^{B}$

In addition to energy, battery charge and discharge rates are also constrained by the effective battery capacity. If it 
takes the battery minimum $\theta^{B}$ hours to charge from zero to full capacity (or, to discharge from full capacity to zero), then charge and discharge rates will be limited according to Riffonneau et al. [16]

$0 \leq \eta^{B} P_{t}^{B^{+}} \leq \frac{C_{t}^{B}}{\theta_{B}}$

$0 \leq \frac{P_{t}^{B^{-}}}{\eta^{B}} \leq \frac{C_{t}^{B}}{\theta_{B}}$

Riffonneau et al. [16] have developed a model for battery cyclic aging, which assumes that battery capacity degradation is linear with respect to battery depth of discharge $(D O D)$ changes:

$C_{t}^{B}-C_{t-1}^{B}= \begin{cases}\zeta^{B} C^{B, \text { nom }}\left[s_{t}^{B}-s_{t-1}^{B}\right], & s_{t}^{B}<s_{t-1}^{B} \\ 0, & s_{t}^{B} \geq s_{t-1}^{B}\end{cases}$

Note that in Eq. $12 s_{t}^{B}$ is ESS state-of-charge $(S O C)$ :

$s_{t}^{B}=\frac{E_{t}^{B}}{C_{t}^{B}}$

If aging is not very significant from one time interval to another, and also over the entire planning horizon, the aging model can be simplified to a linear model:

$\Delta C_{t}^{B}=C_{t}^{B}-C_{t-1}^{B}=-\zeta^{B} \frac{P_{t}^{B^{-}}}{\eta^{B}}$

In addition to aforementioned constraints, EMS needs to limit $P_{t}^{\text {net }}$ in order to prevent excess back injection, and consequent voltage rise:

$f\left(P_{t}^{n e t}, V_{t, i}\right) \leq 0$

\section{Consensus-Based Voltage Regulation}

Ideally, Eq. 15 must be comprised of power flow equations which determine the exact relationship between node real power injections and voltages. However, customers are private entities with limited access to network model and measurements. Therefore, it is almost impossible for them to solve power flow equations.

One solution to this problem is that all customers use fixed constraints on real power feed-in, such as the $70 \%$ rule German utilities apply as we described earlier in the paper [18]:

$P_{t}^{n e t} \geq-0.7 P^{P V, \text { nom }}$

However, a hard limit on real power feed-in may become ineffective when there are multiple PV systems and they are not aware of real power feed-in at other nodes. This problem is especially significant when customers own ESS which adds more flexibility to their net real power exchanges with the grid. If all customers decide to discharge their ESS into the grid when the price of electricity is high, chances are voltage rises beyond the limit along the feeder, even if all customers limit their injection to the maximum allowable value.

If voltage rise occurs along the feeder, all PV+ESSs which are injecting power to the grid are subject to curtailment regardless of their contribution. Since electricity prices and PV generation profiles are similar for all customers located in a neighborhood, it is very probable that their feed-in patterns are almost the same, and therefore in case of overvoltage all of them will incur revenue loss. Hence, it is necessary for customers to "cooperate" with each other in order to keep the voltage under control and to avoid curtailment.

If there exists proper communication infrastructure, each node can acquire information about voltage at other nodes through communication. However, even if each customer knows the exact voltage situation at all other nodes, the information is not much useful, due to the lack of access to the distribution network model. Therefore, collecting voltage at each single node may be inefficient especially in large-scale distribution network spread over a wide geographical area.

Instead, a unique global index that reflects the overall voltage situation may be useful to "estimate" the voltage situation throughout the network. Average voltage deviation (AVD) is a candidate for such an index.

Polymeneas and Benosman [15] have used AVD as a global reference signal fed to the controllers that regulate real power output of DG inverter. A similar index can be used to reach a "consensus" about the voltage situation in the distribution network. AVD, $\delta \bar{V}_{t}$, is defined as:

$$
\begin{aligned}
\delta \bar{V}_{t} & =\sum_{i}^{N}\left(\frac{\delta V_{t, i}}{N}\right) \\
\delta V_{t, i} & =V_{\max }-V_{t, i}
\end{aligned}
$$

Cooperation in order to reach consensus is a classic problem in MAS. Assume that each node in the distribution network is a smart agent capable of:

- Measuring $V_{t, i}$, and $\delta V_{t, i}$

- Communicating with neighboring agents (two-way)

- Running a local EMS engine

If the agents are integrators, with $\mathcal{G}$ as the graph representation of the communication links between them, and $L^{\mathcal{G}}$ as associated graph-Laplacian, in discrete time-domain, a consensus algorithm to reach an agreement on the state of agents can be expressed as [10, 12]:

$x_{k, i}=x_{k-1, i}+\epsilon \sum_{j \in B_{j}}\left(x_{k-1, i}-x_{k-1, j}\right)$ 
The collective dynamics of the network of agents will be:

$\mathbf{x}_{k}=\Phi \mathbf{x}_{k-1}$

When $\mathcal{G}$ is a strongly connected directed graph (digraph) with a maximum degree of $\Delta$, and $0<\epsilon<\frac{1}{\Delta}$, we can show that the agents will reach a consensus, and the consensus value will be $\gamma=\sum_{i}^{N} w_{i} x_{0, i}, \sum_{i}^{N} w_{i}=1$. Also, If the digraph is balanced, an "average" consensus will be reached with $\gamma=\sum_{i}^{N} \frac{x_{0, i}}{N}$.

Therefore, in order to find AVD, agent $i$ in the distribution network 1) measures $\delta V_{k, i}$ at its PCC with the grid, 2) communicates with neighboring agents to send $\delta V_{k, i}$ to them, and receive $\delta V_{k, j}, j \in B_{j}$ from them, and 4) updates its state according to Eq. 19. Step 4 continues until agents reach a consensus on voltage deviation in distribution network.

The convergence of the consensus algorithm depends on the topology of the communication network. In this work, we assume that this topology is identical to that of power distribution network. This ensures strong connectivity of associated communication digraph $L^{\mathcal{G}}$. Also, we can show that the convergence time of consensus algorithm depends on the second smallest eigenvalue of $L^{\mathcal{G}}$ [10], and, it is much smaller than the duration of system scheduling time step $t$ which is an hour in this work.

Knowing AVD alone, cannot provide enough information to determine the exact relationship between voltage fluctuations and real power variations. However, if operating conditions do not vary drastically, agents will eventually learn to associate an AVD to a particular voltage situation. In this work, we assume that agents use the following constraint to react to AVD:

$P_{t}^{n e t} \geq P_{t}^{n e t, \min }$

where $P_{t}^{n e t, \min } \leq 0$ and changes according to Eq. 22

$P_{t}^{n e t, \text { min }}= \begin{cases}P_{t-1}^{\text {net, min }} & \delta \bar{V}_{t} \in \mathrm{N} \\ 0 & \delta \bar{V}_{t} \in \mathrm{H}\end{cases}$

where, $\mathrm{N}$ and $\mathrm{H}$ are Normal and High voltage regions, and are defined accordingly:

- Region $N$ : when $\alpha \Delta V<\delta \bar{V}_{t}<\Delta V$, agents will assume that voltage is normal throughout the distribution network, and they will not change their limits on net injection into the grid.

- Region $H$ : when $0<\delta \bar{V}_{t}<\alpha \Delta V$, agents will assume that voltage is high and stop injecting power into to grid for the next time interval.

$\Delta V=V_{\max }-V_{\text {base }}$, and $V_{\text {base }}$ is the nominal system voltage that is 1 per unit. $\alpha \in(0,1)$ is a scalar that determines the size of voltage regions (see Fig. 2). For example, when

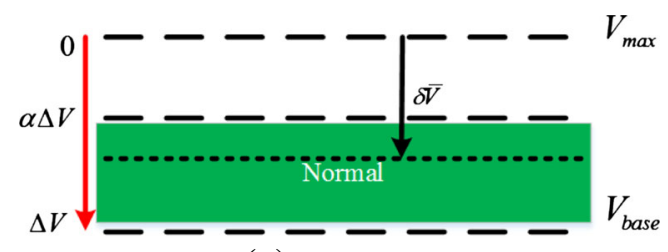

(a) Normal

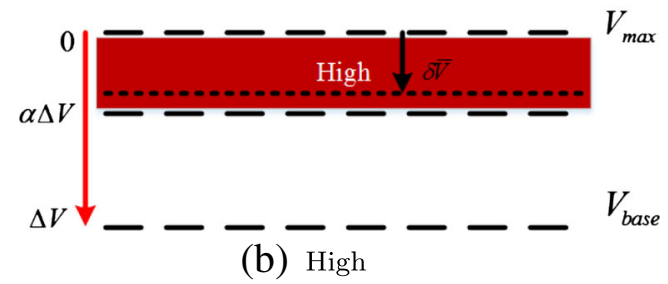

Fig. 2 Voltage regions: a Normal b High

$\alpha=\frac{1}{3}$, it means the size of Regin $H$ is half of the size of Region $N$.

In the definition of voltage regions, we assume that voltage never goes beyond acceptable limits, because if that is the case, all PV+ESSs will face curtailment.

\section{Solution Algorithm}

The EMS engine of each customer needs to solve two problems:

- Primary sizing+scheduling problem

- Pseudo-real-time scheduling problem

The primary sizing+scheduling problem is in fact a planning phase in which EMS determines the size of ESS and the initial power purchase plan (i.e., schedules), whereas the pseudo-real-time scheduling problem intends to determine the power purchase strategy once the voltage situation of distribution network is known.

\section{Primary Sizing+Scheduling Problem}

At this step, customers (i.e. agents) decide about their ESS capacity and their power purchase strategy. They are not willing to share any information about this planning phase with other entities including neighboring agents and the utility. This means that each customer solves the sizing+scheduling problem individually.

The primary sizing+scheduling problem (PSSP) is comprised of Eqs. 1-3, 5-11 and 14, plus the voltage regulatory constraint of Eq. 16. The reason why customers use $70 \%$ rule (16) as the voltage regulatory constraint is obviously lack of information about distribution network model and 


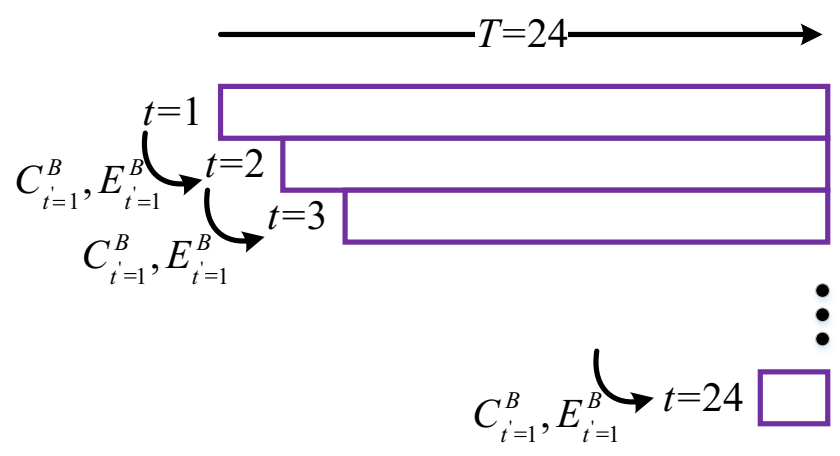

Fig. 3 Shrinking planning horizon

other customers. In other words, Eq. 16 is the only public data about voltage regulation scheme in the distribution network.

Agents solve the optimization problem over planning horizon $T$, which in this work, is 24 hours, or a day ahead. We assume that agents are capable of forecasting their own demand profile, $P_{t}^{D}$, and PV generation, $P_{t}^{P V}$, for the next 24 hours.

The solution to PSSP is the basis for ESS procurement and economic evaluation.

\section{Pseudo Real-Time Scheduling Problem}

After the day-ahead planning, agents need to manage their $P_{t}^{\text {net }}$ in a near real-time fashion to account for voltage conditions. At this step, each agent knows the nominal capacity of ESS, $C^{B, \text { nom }}$, and $P_{t}^{\text {net }}, t=1,2, \ldots, T$ are the only decision variables to determine. The pseudo real-time scheduling problem (PRSP) includes (1), (3)-(7), (9)-(11), and (14), as well as voltage regulatory constraints (21) and (22). The initial condition on energy (8) does not need to be considered in PRSP as will be further discussed.

Unlike PSSP, agents cannot solve PRSP as a whole over the entire planning horizon $T$, because voltage situation changes in real-time, and consequently, voltage regulatory constraints (21) and (22) need to be updated at each time step. On the other hand, due to ESS dynamic behavior and aging, PRSP cannot be solved at each time step independently of other time steps.

In order to be able to update and solve PRSP at each time interval, agents use the following algorithm:

1. For the first time interval $(t=1)$ agents purchase power from the grid according to the solution of PSSP.

2. Close to the end of time interval, agents communicate with their neighbors and exchange $\delta V_{t}$. After agents reach a consensus about the voltage situation in the distribution network, they update $P_{t}^{\text {net, } m i n}$ for the next time interval according to Eq. 22.

3. When the first time interval passes, planning horizon reduces to the remaining hours ahead $(t=2,3, \ldots, T)$. For the next time interval, agents rerun PRSP over the shrunken planning horizon $t^{\prime}=1,2,3, \ldots T^{\prime}, T^{\prime}=$ $T-1$ with updated voltage regulatory constraints. Also, initial conditions on ESS capacity and energy will be changed to $C_{t^{\prime}=1}^{B}=C_{t^{=1}}^{B}$ and $E_{t^{\prime}=1}^{B}=E_{t=1}^{B}$. This means that the effective capacity and available energy of ESS at the beginning of each time interval are equal to those of the end of the previous time interval. These recursive initial conditions, ensure that PRSP preserves the dynamic nature of ESS.

4. Steps 3 and 4 continue until the end of the planning horizon.

Figure 3 shows how the planning horizon shrinks and initial conditions are transferred from previous time step to current one.

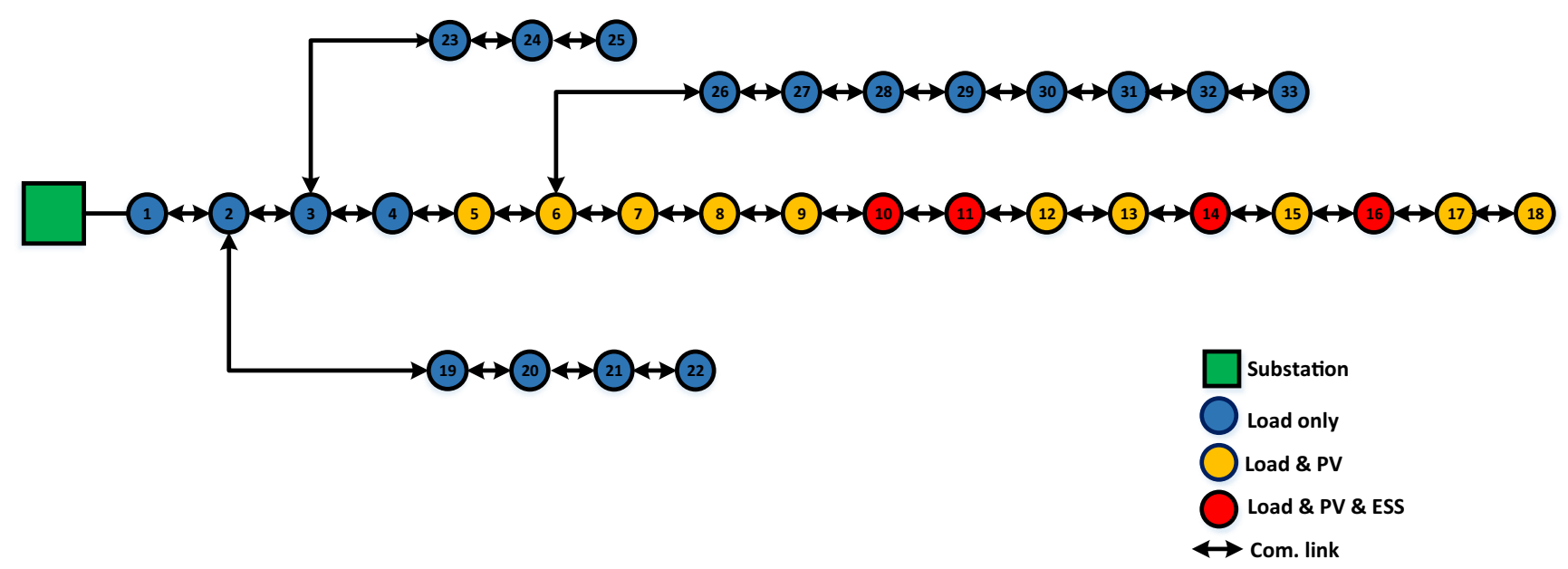

Fig. 4 Modified IEEE 33-bus test feeder 


\section{Simulation Results}

In this section we analyze the performance of the proposed distributed energy management scheme for PV+ESS. Figure 4 shows IEEE 33-bus feeder that we use as the test system in this study [14].

We modify the feeder to include PV generation at nodes 5 to 18 on the main branch (encompassing nodes 1 to 18 ). $\mathrm{PV}$ penetration is $31 \%$ of the feeder's total real power. For each of these nodes, installed PV capacity is equal to the peak real power demand at that node. Also, note that the feeder loading is modified from the original test feeder (See Appendix). We assume that there are two-way communication links between each two neighboring nodes, and the

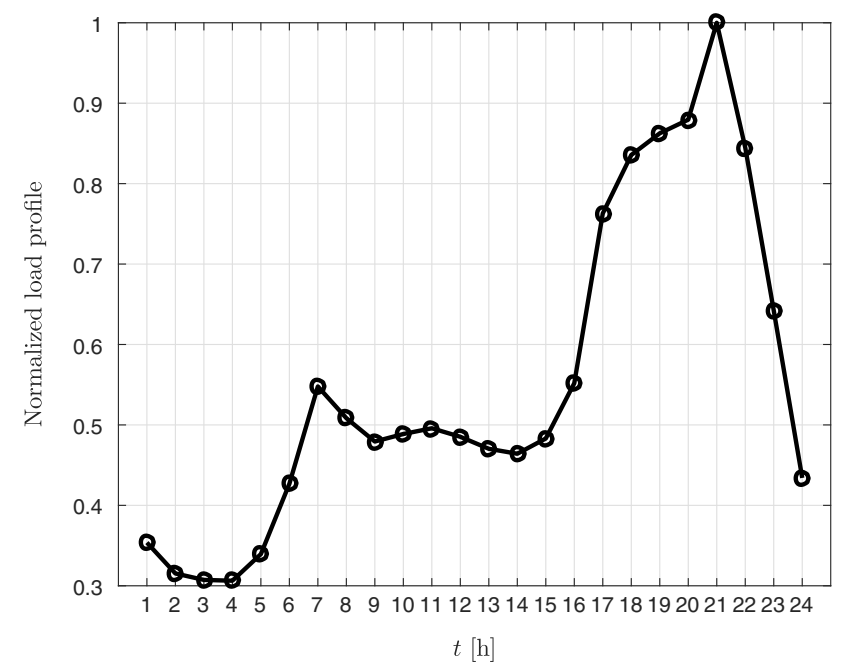

(a) Normalized load profile

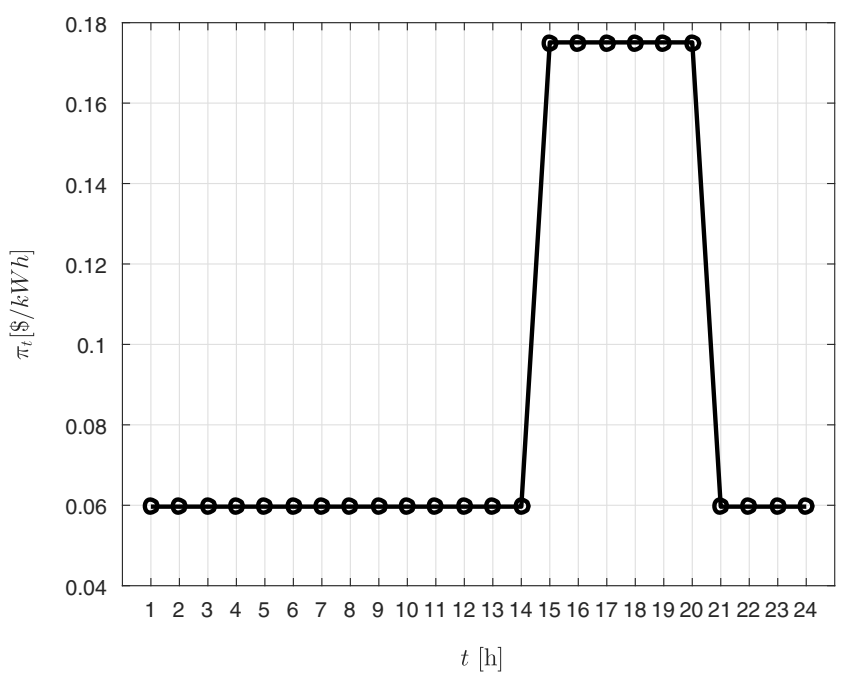

(c) Time-of-use pricing topology of the communication network is identical to that of the electrical distribution network. To generate daily load and PV generation profiles we assume that real and reactive power demand at each node varies according to the normalized daily load profile shown in Fig. 5a [13], and PV generation varies according to the normalized profile shown in Fig. 5b [11]. Moreover, we assume that the utility uses a two-level time-of-use pricing mechanism shown in Fig. 5c [20]. Figure 5d shows daily voltage profiles of nodes 10 to 18 (calculated by MATPOWER [24]) when there is no voltage regulation. During hours 11 to 13 voltage at nodes 13 to 18 goes above the allowable limit of 1.058 p.u., that is the maximum allowable voltage during extreme conditions [19]. All customers with PV generation will face

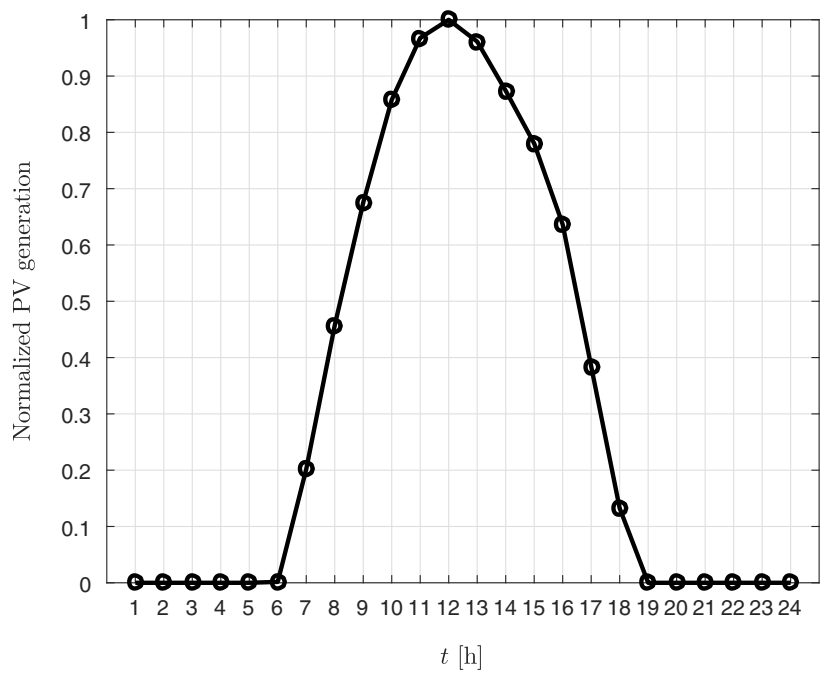

(b) Normalized PV generation profile

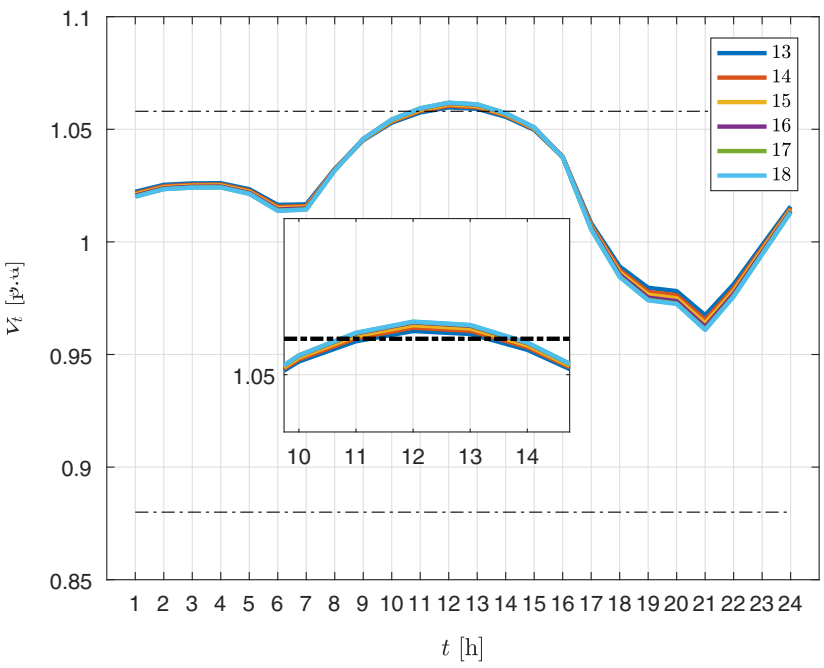

(d) Voltage profile w/o regulation

Fig. 5 System data 


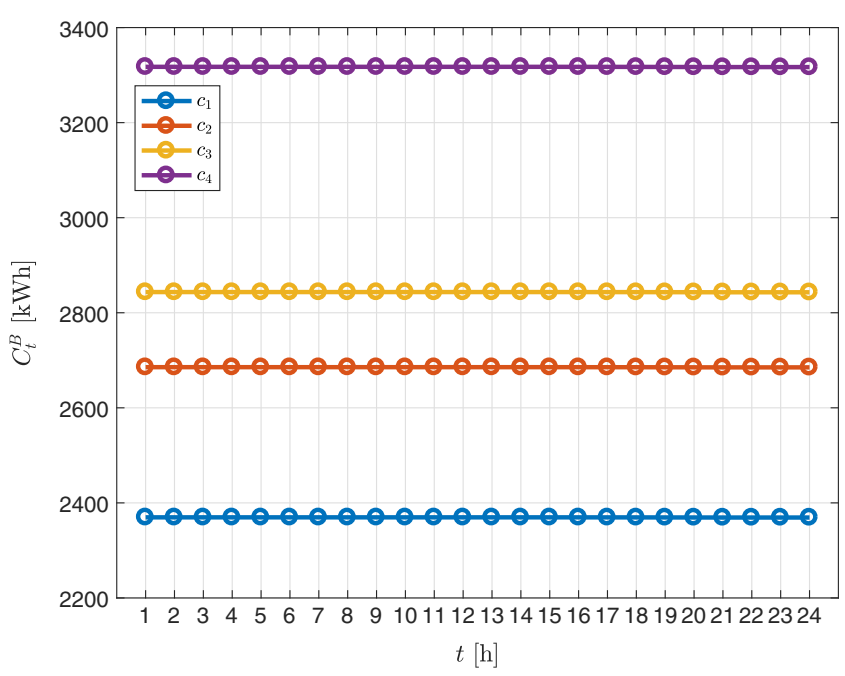

(a) ESS effective capacity

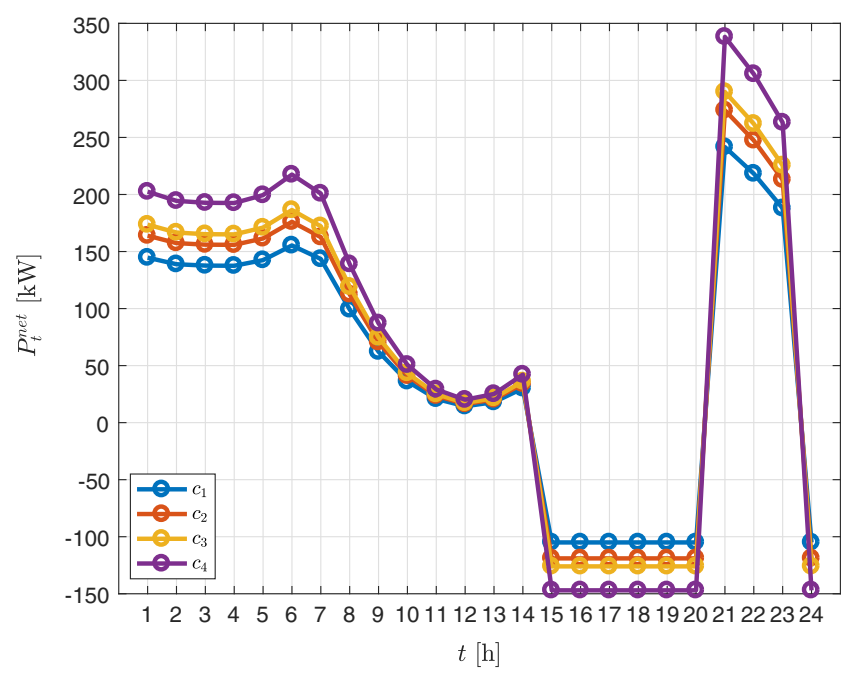

(c) Net real power purchase

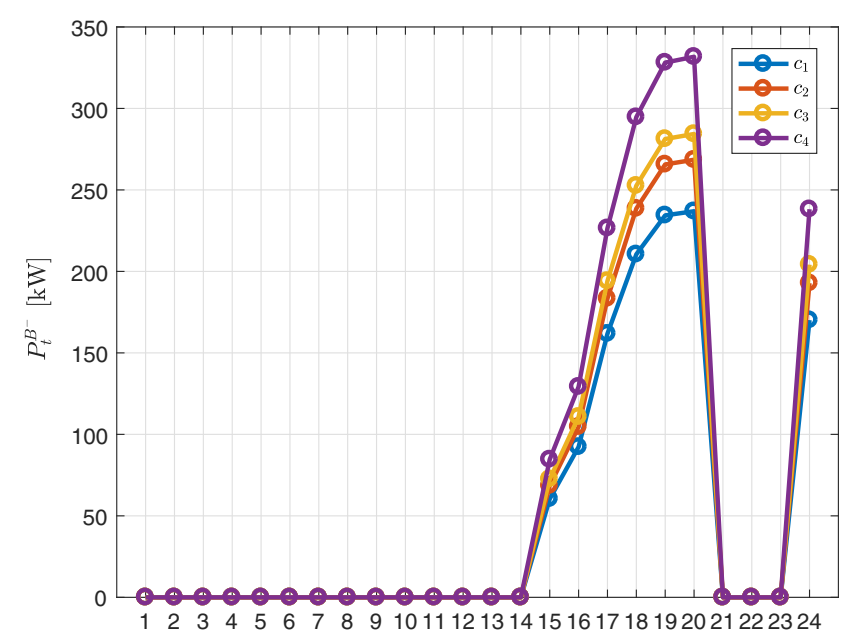

(e) ESS discharge rate

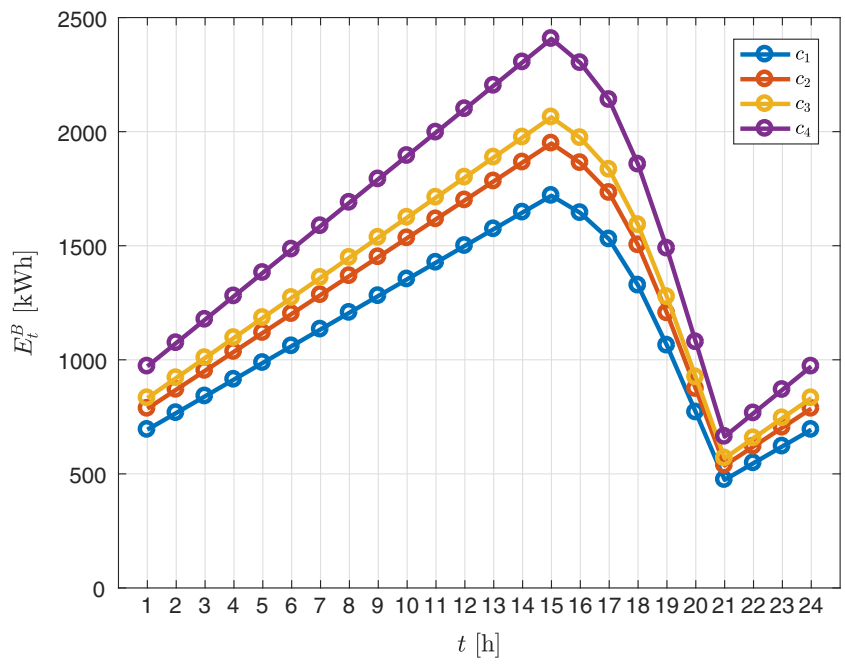

(b) ESS energy

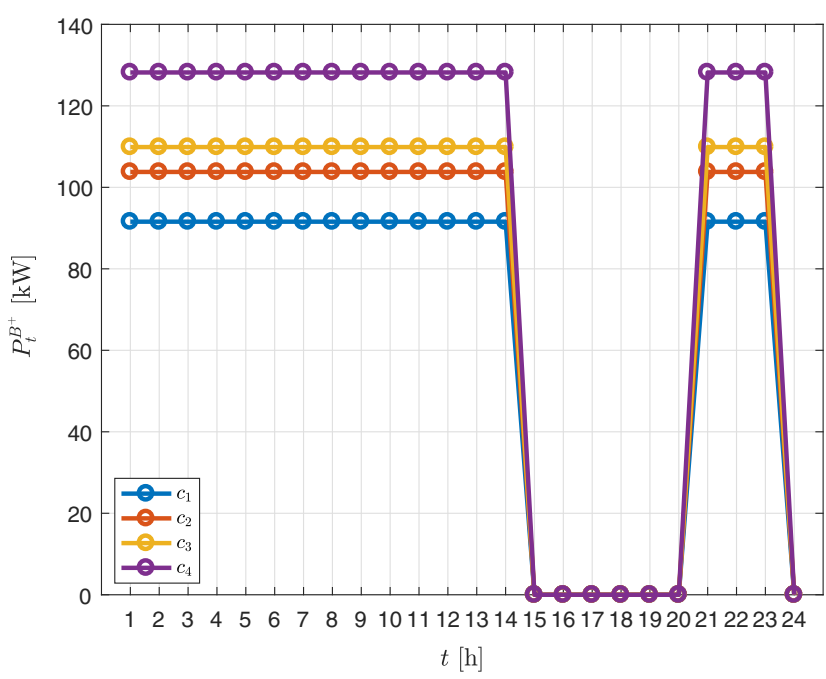

(d) ESS charge rate

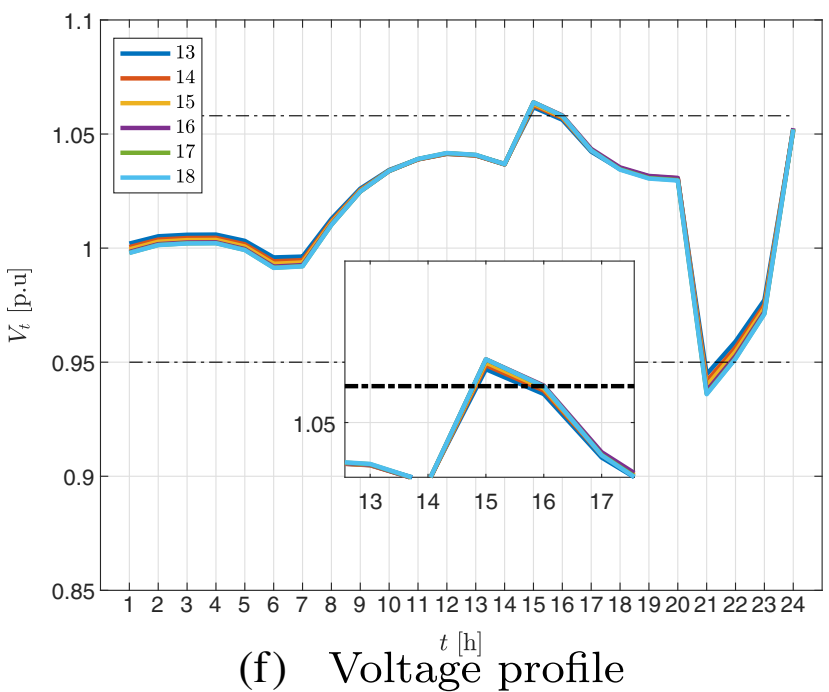

Fig. 6 Simulation results for PSSP 
curtailment during these hours. We assume that when PV generation is non-dispatchable, and thus curtailment means the PV system must be completely shut off. Customers at nodes $10,11,14$, and 16 (from now on, customers 1-4) with respective real peak demand of 150, 170, 180 and 210 [kW] and installed PV capacity equal to the real peak demand, plan to install and use ESS to avoid curtailment.

\section{Primary Sizing+Scheduling}

Figure 6 shows the solution of PSSP for customers 1-4, and 16. PSSP is a non-linear programming problem, we use MATLAB optimization toolbox to determine the solution to it. Nominal ESS capacities are 2370, 2686, 2846, and $3317[\mathrm{kWh}$ ] respectively. Note that $\lambda=120 \$ / \mathrm{kWh}$, $\theta^{B}=8 \mathrm{~h}, \zeta^{B}=3 \times 10^{-4}, \eta^{B}=80 \%$, and $d^{B}=20 \%$. Customers charge their ESS during off-peak hours when the electricity is cheap; whether or not there is PV generation. This means that they are arbitraging energy and charging their ESS from the grid in the absence of PV generation. Significant ESS discharge occurs during hours 15-20 when the electricity price and demand are at peak. The trajectory of net power purchase from the grid (Fig. 6c) shows that during hours 15-20 when ESSs discharge significantly, $P_{t}^{\text {net }}=P_{t}^{\text {net }, \text { min }}$ or $70 \%$ of installed PV capacity. Figure $6 f$ shows that voltage at nodes 13-18 goes above the maximum allowable limit during hour 15 . This is despite the fact that PV+ESSs located at nodes 10,11, 14 and 16 have limited their net feed-in to $70 \%$ of installed PV capacity, and other PV nodes (without ESS) have shut down their generating system during this hour. We can conclude that a fixed limit

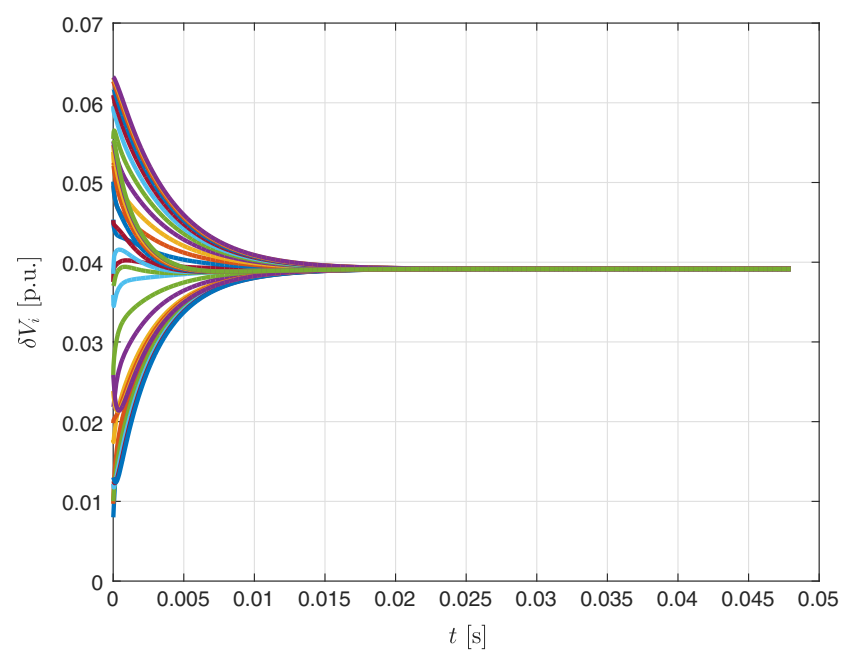

Fig. 7 Consensus dynamics on net injection cannot fully regulate the voltage when there are multiple PV+ESS on the feeder.

\section{Pseudo Real-Time Scheduling}

In PRSP, customers start off with the results of PSSP stage. Then, for the next hours ahead they communicate with each other to reach a consensus about AVD in the distribution network, and manage their net power purchases accordingly. Therefore, for $t=1$ PRSP and PSSP are the same. From Fig. 4, we can see that the maximum degree of digraph $\Delta=3$ and hence $\epsilon<\frac{1}{3}$. For $\alpha=0.336$, Fig. 8 shows the simulation results for the PRSP case. For illustration, Fig. 7 shows how agents reach a consensus about AVD at the end of first time step $(t=1)$. The convergence time is about 0.04 $\mathrm{s}$ which is significantly smaller than the duration of scheduling time step (1h). Compared to the PSSP case, customers 1-4 keep their feed-in level at 70\% of installed PV capacity only for hours 16 and 19, which clearly shows how cautious agents become when voltage starts raising. As Fig. $8 \mathrm{f}$ shows, in this case, no overvoltage happens in the distribution network over the entire planning horizon. Selection of $\alpha$ plays a significant role in the voltage regulation process. There is a trade-off between voltage regulation capability and economic benefits. Smaller $\alpha$ s may lead to poor voltage regulation, while larger $\alpha$ brings less financial benefits. Proper selection of $\alpha$ can happen through learning, assuming that operating conditions of the distribution network do not change drastically over time.

\section{Economic Analysis}

\section{4-Hour Planning Horizon}

Table 1 shows the daily electricity bill of customers 1-4 for four different cases: No ESS, PSSP, PRSP with $\alpha=0.431$, and PRSP with $\alpha=0.336$. For the no ESS and PSSP cases, if voltage goes above 1.058 p.u. all PV nodes (PV only and PV+ESS nodes) will shut down their PV systems. As aforementioned, selection of $\alpha$ has a significant impact on the profitability of ESS. PRSP with $\alpha=0.336$ has the smallest total electricity costs among all cases. Also, all the cases with ESS have less total electricity costs compared to case without ESS.

Customers 1-4 have paid \$284400, \$322320, \$341520, and $\$ 398040$ to purchase and install their ESSs. Although cost savings on the customers' electricity bills sound significant, ESS is subject to degradation and has a limited lifetime. In the following section, we determine the lifetime of ESSs and run an economic assessment to see whether or not ESS technology is justifiable for customers. 


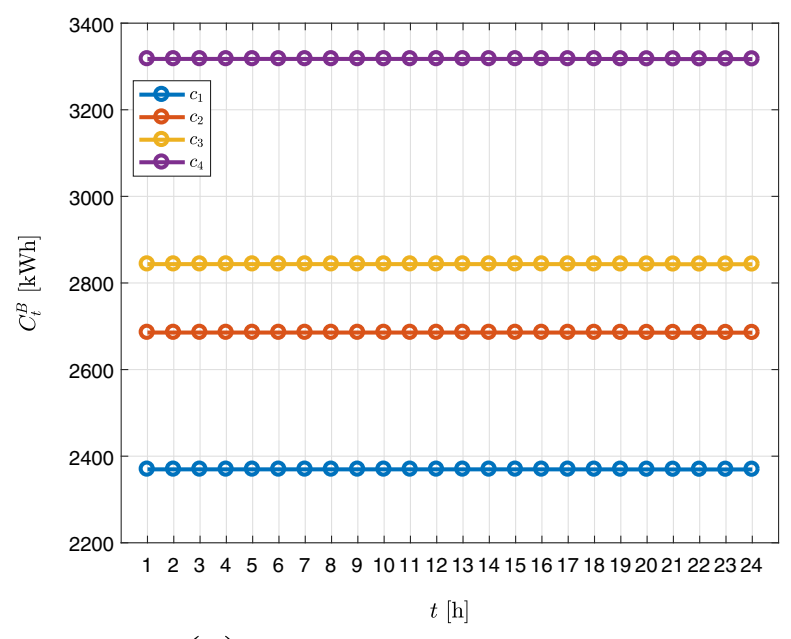

(a) ESS effective capacity

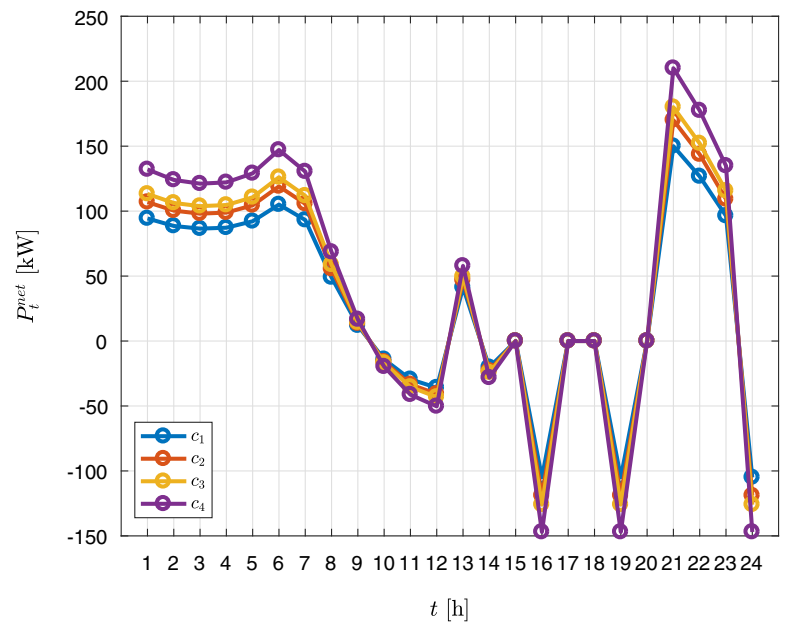

(c) Net real power purchase

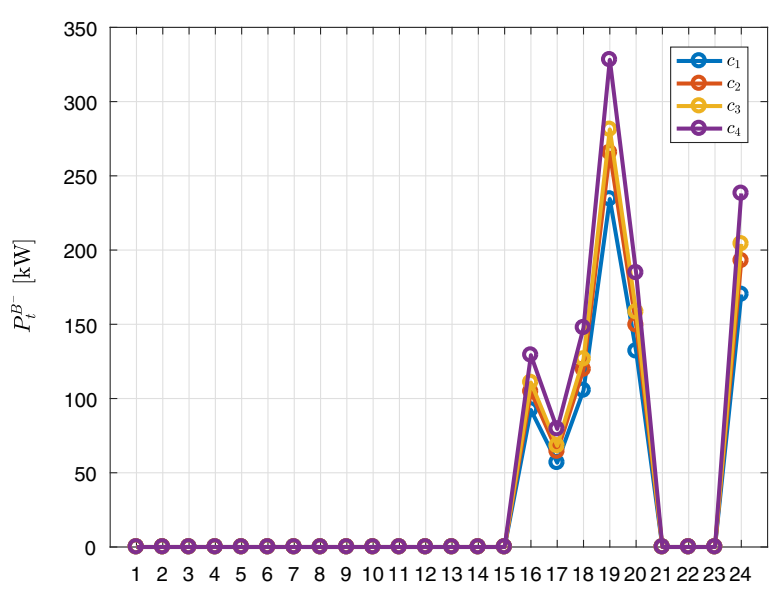

(e) ESS discharge rate

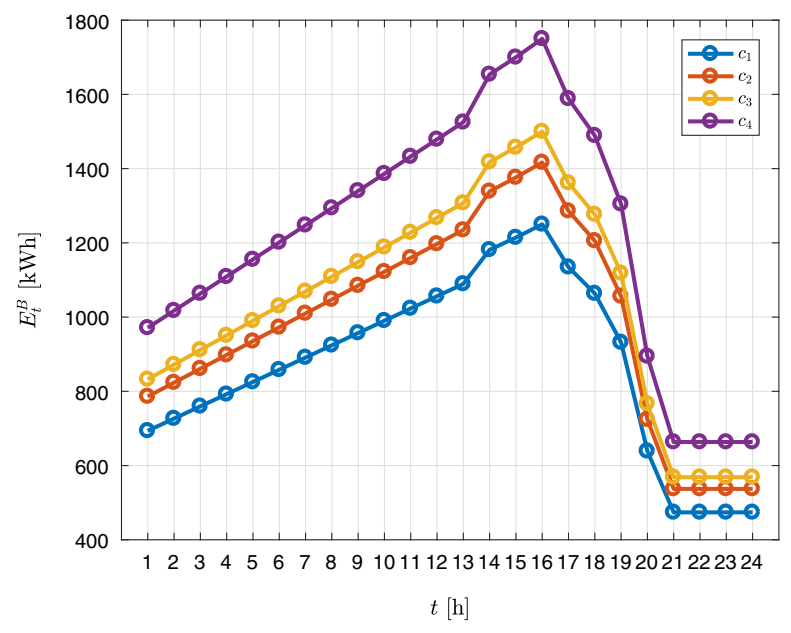

(b) ESS energy

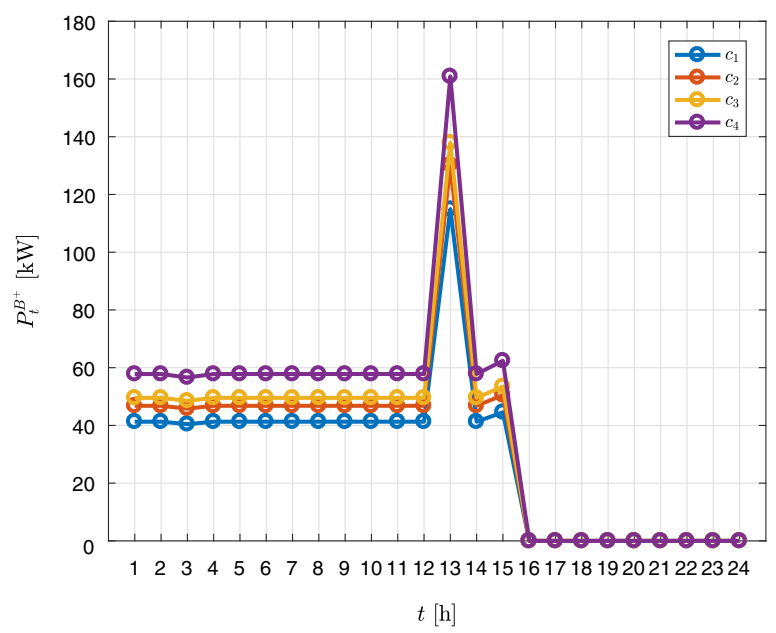

(d) ESS charge rate

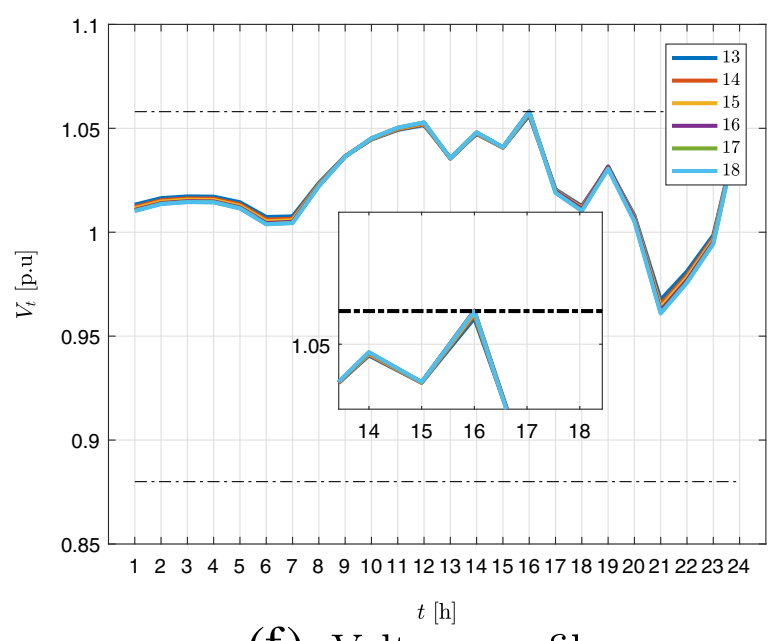

(f) Voltage profile

Fig. 8 Simulation results for PRSP 
Table 1 Economic analysis for a 24-hour planning horizon

\begin{tabular}{|c|c|c|c|c|c|c|c|c|c|c|c|c|c|c|c|c|}
\hline \multirow{2}{*}{$\begin{array}{l}\text { Case } \\
\text { Customer }\end{array}$} & \multicolumn{4}{|c|}{ Bill [\$] } & \multicolumn{4}{|c|}{ Degradation [\$] } & \multicolumn{4}{|c|}{ Total [\$] } & \multicolumn{4}{|c|}{ Savings on bill[ $[\$]$} \\
\hline & 1 & 2 & 3 & 4 & 1 & 2 & 3 & 4 & 1 & 2 & 3 & 4 & 1 & 2 & 3 & 4 \\
\hline w/o ESS & 116 & 132 & 140 & 163 & 0 & 0 & 0 & 0 & 116 & 132 & 140 & 163 & 0 & 0 & 0 & 0 \\
\hline PSSP & 62 & 71 & 75 & 87 & 42 & 48 & 50 & 59 & 104 & 118 & 125 & 146 & 54 & 61 & 65 & 76 \\
\hline$\alpha=0.431$ & 36 & 40 & 43 & 50 & 21 & 24 & 26 & 30 & 57 & 65 & 68 & 80 & 81 & 92 & 97 & 113 \\
\hline$\alpha=0.336$ & 18 & 20 & 22 & 25 & 28 & 32 & 34 & 40 & 46 & 53 & 56 & 65 & 98 & 112 & 118 & 138 \\
\hline
\end{tabular}

\section{Yearly Planning Horizon}

To determine the lifetime of ESS, we use the method proposed in [2]. In this approach, the PRSP problem is solved iteratively, until the ESS dies. In each iteration, initial condition (3) is updated such that the nominal capacity, $C^{B, n o m}$, is equal to the effective capacity at the end of the previous day; i.e., $C_{T}^{B}$ of the previous iteration. ESS is considered to be dead when the effective capacity reaches $60 \%$ of the installed capacity.

Figure 9a shows how ESSs degrade in time. The lifetime of all ESSs are calculated to be 12 years. Figure $9 \mathrm{~b}$ shows how yearly cost savings on the electricity bill change over the lifetime of each ESS. Considering the interest rate of $0.5 \%$, we can calculate that customers $1-4$ will respectively obtain $\$ 11600, \$ 13152, \$ 13858, \$ 16536$ of financial benefit if they install ESS.

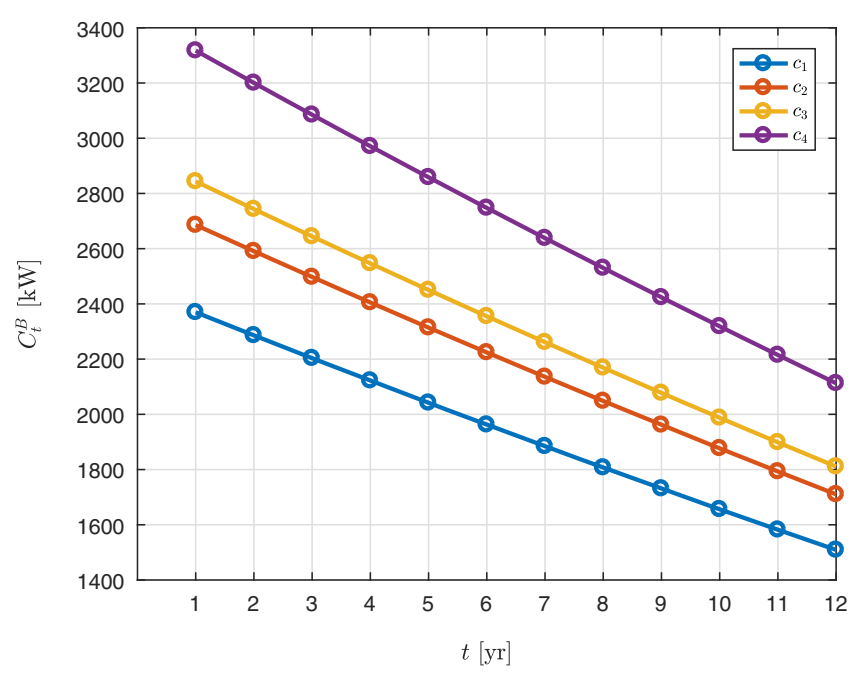

(a) Capacity degradation over lifetime

\section{Conclusion}

We developed a method of sizing and managing privatelyowned ESS in a distribution network with high PV penetration considering voltage rise mitigation. First, private entities (customers) size the ESS to be installed in parallel with their PV system, with a hard limit on real power feed-in being the voltage regulatory constraint. Second, a consensus-based EMS will manage net power exchanges with the grid in a pseudo real-time fashion to account for voltage conditions. Smart nodes in the distribution network cooperatively share voltage deviation measured at their own PCC with the neighboring nodes. Consensus on the average voltage deviation will be the basis for updating the voltage regulatory constraint. Simulation results show that the proposed method can effectively mitigate voltage rise issue, while reducing customers' electricity bill. In addition, we

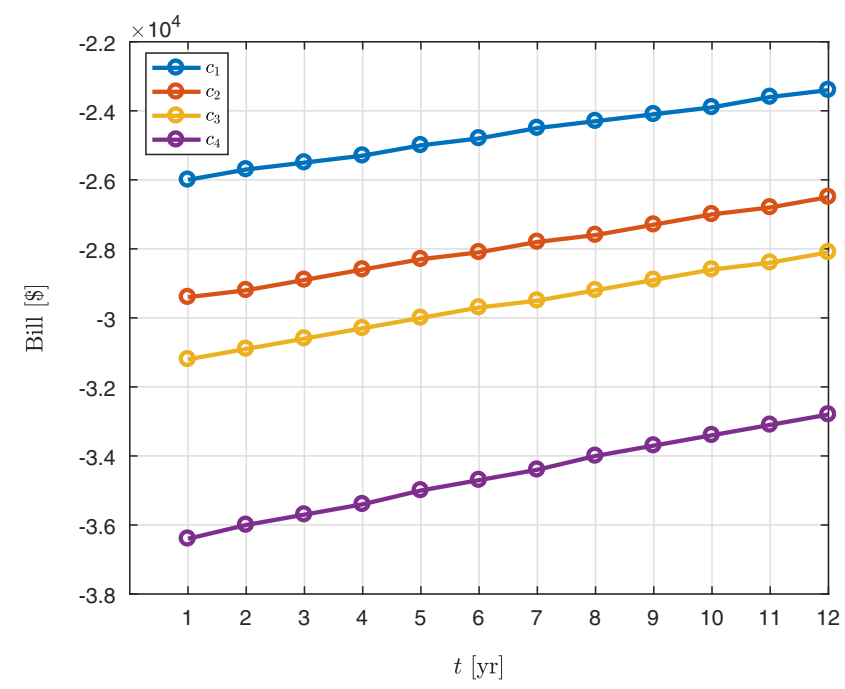

(b) Profit over lifetime

Fig. 9 Economic analysis over lifetime 
developed an iterative method to determine the lifetime of ESS and studied the economy of PV+ESS over the lifetime to ensure the economic viability of ESS.

\section{Appendix}

Table 2 Appendix: Load data

\begin{tabular}{|c|c|c|}
\hline Node & Real Power $[\mathrm{kW}]$ & Reactive Power [kVar] \\
\hline 1 & 0 & 0 \\
\hline 2 & 100 & 60 \\
\hline 3 & 90 & 40 \\
\hline 4 & 100 & 80 \\
\hline 5 & 40 & 30 \\
\hline 6 & 40 & 20 \\
\hline 7 & 50 & 50 \\
\hline 8 & 50 & 50 \\
\hline 9 & 40 & 20 \\
\hline 10 & 150 & 10 \\
\hline 11 & 170 & 20 \\
\hline 12 & 40 & 40 \\
\hline 13 & 40 & 40 \\
\hline 14 & 180 & 20 \\
\hline 15 & 50 & 20 \\
\hline 16 & 210 & 50 \\
\hline 17 & 30 & 30 \\
\hline 18 & 30 & 30 \\
\hline 19 & 90 & 40 \\
\hline 20 & 90 & 40 \\
\hline 21 & 90 & 40 \\
\hline 22 & 90 & 40 \\
\hline 23 & 90 & 50 \\
\hline 24 & 420 & 200 \\
\hline 25 & 420 & 200 \\
\hline 26 & 60 & 25 \\
\hline 27 & 60 & 25 \\
\hline 28 & 60 & 20 \\
\hline 29 & 120 & 70 \\
\hline 30 & 200 & 600 \\
\hline 31 & 150 & 70 \\
\hline 32 & 210 & 100 \\
\hline 33 & 60 & 40 \\
\hline
\end{tabular}

\section{References}

1. Akhil AA, Huff G, Currier AB, Kaun BC, Rastler DM, Chen SB, Cotter AL, Bradshaw DT, Gauntlett WD (2013) DOE/EPRI 2013 electricity storage handbook in collaboration with NRECA . Sandia National Laboratories Albuquerque, Albuquerque, pp 19-20

2. Ansari B, Shi D, Sharma R, Simoes M (2016) Economic analysis, optimal Sizing and management of energy storage for PV grid integration. In: IEEE T\&D Conference \& Exposition, IEEE, pp 1-5. IEEE

3. Baran ME, El-Markabi IM (2007) A multiagent-based dispatching scheme for distributed generators for voltage support on distribution feeders. IEEE Trans Power Syst 22(1):52-59

4. Broderick RJ (2012) Managing high PV deployment on the EPS: Overview of key challenges and mitigation options, Technical report, Sandia National Laboratories

5. DOE GridEnergyStorage Technical report, U.S. Department of Energy

6. Ellis A, Nelson R, Von Engeln E, Walling R, McDowell J, Casey L, Seymour E, Peter W, Barker C, Kirby B (2012) Reactive power interconnection requirements for PV and wind plantsrecommendations to NERC. Sandia National Laboratories, Albuquerque, p 87185

7. Hashemi S, Ostergaard J, Yang G (2014) A scenario-based approach for energy storage capacity determination in LV grids with high PV penetration. IEEE Trans Smart Grid 5(3):1514-1522

8. Joo JY, Ilic MD (2013) Multi-layered optimization of demand resources using lagrange dual decomposition. IEEE Trans Smart Grid 4(4):2081-2088. https://doi.org/10.1109/TSG.2013.2261565

9. Liu Y, Bebic J, Kroposki B, De Bedout J, Ren W (2008) Distribution system voltage performance analysis for high-penetration PV. In: IEEE Energy 2030 Conference, IEEE, pp 1-8. IEEE

10. Mesbahi M, Egerstedt M (2010) Graph theoretic methods in multiagent networks. Princeton University Press, Princeton, pp 42-71

11. NREL, http://www.nrel.gov/electricity/transmission/solar_integration_ methodology.html, 2016. Online

12. Olfati-Saber R, Fax A, Murray RM (2007) Consensus and cooperation in networked multi-agent systems. Proc IEEE 95(1):215-233

13. OpenEI, http://www.nrel.gov/electricity/transmission/solar_integration methodology.html, 2016. Online

14. Parasher R (2014) Load flow analysis of radial distribution network using linear data structure. arXiv: 1403.4702

15. Polymeneas E, Benosman M (2014) Multi-agent coordination of DG inverters for improving the voltage profile of the distribution grid. In: IEEE PES General Meeting Conference \& Exposition, IEEE, pp 1-5. IEEE

16. Riffonneau Y, Bacha S, Barruel F, Ploix S (2011) Optimal power flow management for grid connected PV systems with batteries. IEEE Trans Sustainable Energy 2(3):309-320

17. Sulc P, Backhaus S, Chertkov M (2014) Optimal distributed control of reactive power via the alternating direction method of multipliers. IEEE Trans Energy Convers 29(4):968-977

18. von Appen J, Stetz T, Braun M, Schmiegel A (2014) Local voltage control strategies for PV storage systems in distribution grids. IEEE Trans Smart Grid 5(2):1002-1009

19. Wang Y, Zhang P, Li W, Xiao W, Abdollahi A (2012) Online overvoltage prevention control of photovoltaic generators in microgrids. IEEE Trans Smart Grid 3(4):2071-2078

20. Xcel, https://www.xcelenergy.com/staticfiles/xe/Regulatory/, 2016. Online

21. Yang Y, Li H, Aichhorn A, Zheng J, Greenleaf M (2014) Sizing strategy of distributed battery storage system with high penetration of photovoltaic for voltage regulation and peak load shaving. IEEE Trans Smart Grid 5(2):982-991

22. Yu L, Czarkowski D, De León F (2012) Optimal distributed voltage regulation for secondary networks with DGs. IEEE Trans Smart Grid 3(2):959-967

23. Zhang B, Lam AY, Domínguez-García AD, Tse D (2015) An optimal and distributed method for voltage regulation in power distribution systems. IEEE Trans Power Syst 30(4):1714-1726

24. Zimmerman RD, Murillo-Sánchez CE, Thomas RJ (2011) Matpower: Steady-state operations, planning, and analysis tools for power systems research and education. IEEE Trans Power Syst 26(1):12-19 